\title{
Probing the X-ray Variability of X-ray Binaries
}

\author{
Wenfei Yu \\ Astronomical Institute "Anton Pannekoek", University of Amsterdam, \\ Kruislaan 403, 1098 SJ, Amsterdam, The Netherlands \\ Institute of High Energy Physics, Beijing, China (previous address)
}

\begin{abstract}
Kilohertz quasi-periodic oscillations (kHz QPOs) has been regarded as representing the Keplerian frequency at the inner disk edge in the neutron star X-ray binaries. The so-called "parallel tracks" on the plot of the $\mathrm{kHz}$ QPO frequency vs. X-ray flux in neutron star Xray binaries, on the other hand, show the correlation between the $\mathrm{kHz}$ QPO frequency and the X-ray flux on time scales from hours to days. This is suspected as caused by the variations of the mass accretion rate through the accretion disk surrounding the neutron star. We show here that by comparing the correlation between the $\mathrm{kHz}$ QPO frequency and the X-ray count rate on a certain QPO time scale observed approximately simultaneous in the Fourier power spectra of the X-ray light curve, we have found evidences that the X-ray flux of millihertz QPOs in neutron star X-ray binaries is generated inside the inner disk edge if adopting that the kilohertz QPO frequency is an orbital frequency at the inner disk edge.
\end{abstract}

\section{Introduction}

Kilohertz quasi-periodic oscillations ( $\mathrm{kHz}$ QPOs) in the X-ray flux of about 20 neutron star low mass X-ray binaries (LMXBs) (see van der Klis 2000 for a recent review) are suggested to be the indicator of the orbital frequency at the inner disk edge (Strohmayer et al. 1996; Miller, Lamb \& Psaltis 1998; Titarchuk, Lapidus \& Muslimov 1998; Stella, Vietri \& Morsink 1999;). One of the evidence for this, in addition to the millisecond time scales themselves, is that there are correlations between the $\mathrm{kHz}$ QPO frequency and the X-ray flux in these neutron star X-ray binaries on time scales from hours to days (e.g. Yu et al. 1997; Mendez et al. 1998; Zhang et al. 1998). Especially in the two neutron star soft X-ray transients (SXTs) 4U 1608-52 and Aquila X-1, the correlation holds in a large flux range up to an order of magnitude (Mendez 1999/astroph/9903469). The correlation forms the so-called "parallel tracks" in the plot of $\mathrm{kHz}$ QPO frequency vs. X-ray flux in "atoll" sources. For those "Z" sources the correlation in the plot of $\mathrm{kHz}$ QPO frequency vs. X-ray flux may appear as negative correlation, depending on the source branch it stays (Wijnands et al. 1997; Yu, van der Klis \& Jonker 2001). These seem consistent with the general idea that an increase of the mass accretion rate (thus an increase/decrease of the X-ray flux) will cause the inner disk edge at radius $R_{i n}$ approach closer to 
the neutron star, which would introduce an increase of the orbital frequency $\nu_{O}$ at the inner disk edge, and vise versa. The response of the inner disk radius to the mass accretion rate through the disk has been widely accepted in both theory and observations.

Under the assumption that the source movements on those correlation tracks are because of the variation of the mass accretion rate at the inner disk, and the $\mathrm{kHz}$ QPO frequency is an indicator of the orbital frequency at the inner disk edge (notice that the $\mathrm{kHz}$ QPO frequency is not necessary to be the orbital frequency itself), the correlation between the $\mathrm{kHz}$ QPO frequency and the X-ray flux on each correlation track then probably represents the correlation between the inner disk radius and the mass accretion rate through the inner disk. Thus the X-ray flux variabilities on each track which occurs on time scales of hours to days could be an accretion mode. Similar variability modes on shorter time scales of accretion origin will probably introduce the same correlation between the inner disk radius and the X-ray count rate on its time scale. This then leads to the same correlation between the $\mathrm{kHz}$ QPO frequency and the X-ray count rate as that on the long-term tracks, but on a shorter time scale. Usually the $\mathrm{X}$-ray variability of the neutron star X-ray binaries contain a few simultaneous variability components, e.g. continuous noise components and QPOs, covering a large range of time scales from $\mathrm{mHz}$ to $\mathrm{kHz}$. The correlation between the $\mathrm{kHz}$ QPO frequency and the X-ray count rate tracking a certain variability component then could be a diagnostic of the variability mode and answer whether it is the same as that forms the long-term correlation tracks.

\section{Observations and Results}

The "parallel tracks" are usually observed in those "atoll" sources such as 4U 1608-52 (Yu et al. 1997; Mendez et al. 1998), Aquila X-1 (Zhang et al. 1998; Mendez et al. 1999), and $4 \mathrm{U}$ 1728-34 (Mendez \& van der Klis 1999). In those "Z" sources, the correlation between the $\mathrm{kHz}$ QPO frequency and the X-ray count rate is complicated. For example, on the Normal Branch (NB) of Sco X-1, the $\mathrm{kHz}$ QPO frequency appears anti-correlated with the X-ray count rate, but correlated to the inferred mass accretion rate (Yu, van der Klis \& Jonker 2001).

\section{1. $\mathrm{mHz}$ QPO in the "Atoll" source 4U 1608-52}

$4 \mathrm{U} 1608-52$ is an X-ray burster and a soft X-ray transient (SXT). In the observations of its outburst decay by the Rossi X-ray timing explorer, $\mathrm{kHz}$ QPOs were observed showing its frequency correlated with the X-ray count rate on the time scales of hours to days but not longer (eg. Yu et al. 1997; Mendez et al. 1998). More recently, simultaneous with the $\mathrm{kHz}$ QPOs, millihertz QPOs at a frequency of $7-9 \times 10^{-3} \mathrm{~Hz}$ in the X-ray flux of $4 \mathrm{U} 1608-52$ were observed. They are associated with the soft X-ray photons below $5 \mathrm{keV}$ and probably have close relationship with the type I X-ray bursts. This leads to the speculation that the $\mathrm{mHz}$ QPO is caused by a mode of nuclear burning at the neutron star surface (Revnivtsev et al. 2001). The corresponding $\mathrm{kHz}$ QPO frequency evolution in $4 \mathrm{U} 1608-52$ in response to the $\mathrm{mHz}$ pulses was studied with a peak-aligned method after removing the long-term trend by Yu \& van der Klis (2002). They found that the $\mathrm{kHz}$ QPO frequency is anti-correlated with the $\mathrm{mHz}$ QPO flux 


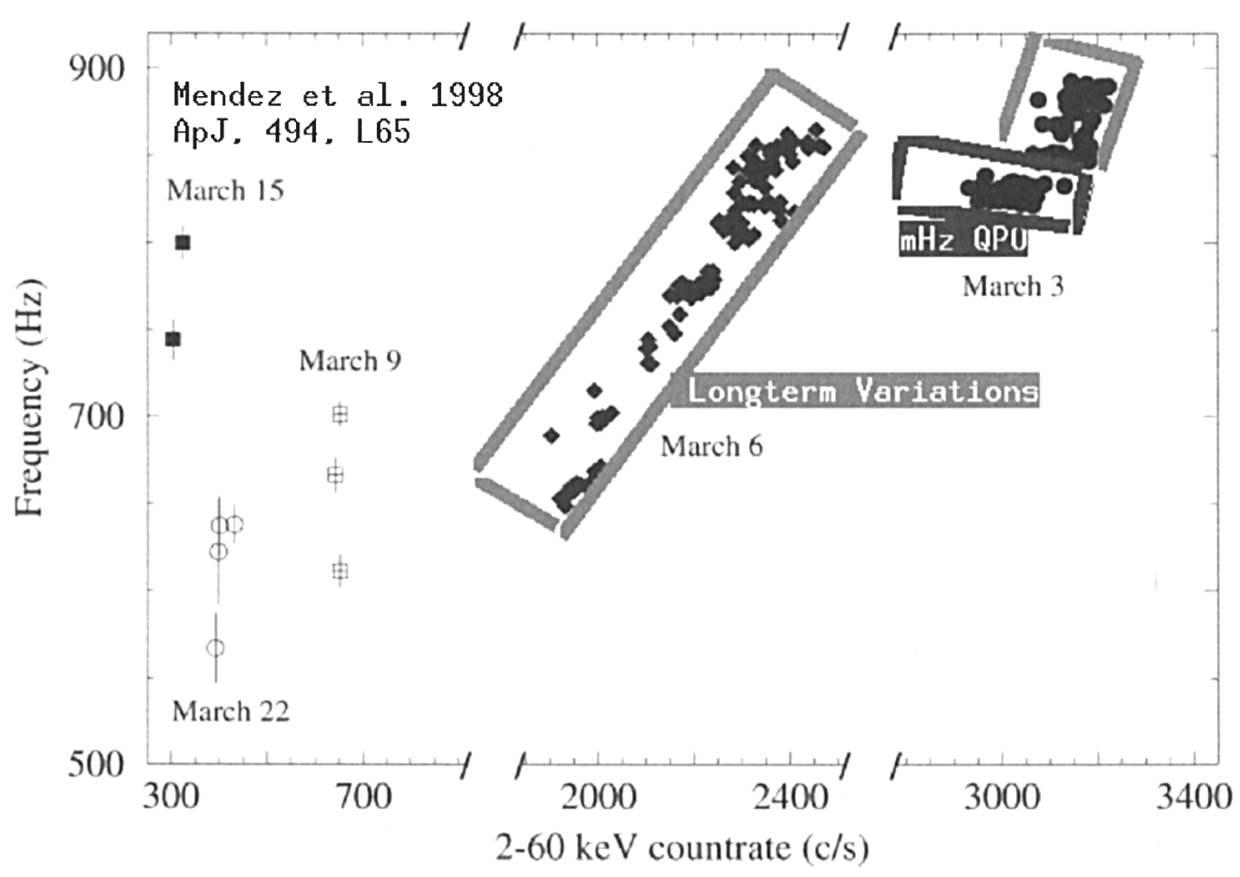

Figure 1. The positive correlations between the $\mathrm{kHz}$ QPO frequency and the X-ray count rate on long time scales and on short time scales corresponding to $\mathrm{mHz}$ QPOs in $4 \mathrm{U}$ 1608-52 (see also Yu \& van der Klis 2002).

which can be tracked by the average $\mathrm{mHz}$ QPO count rate of 12 seconds. The $\mathrm{kHz}$ QPO frequency shift in response to the $\mathrm{mHz}$ QPOs is about $0.6 \mathrm{~Hz}$.

\subsection{Variability modes in the plot of $\mathrm{kHz}$ QPO frequency vs. X-ray count rate}

To compare the X-ray count rate variation on a certain QPO time scales with that on longer time scales which forms "parallel" tracks in the plot of $\mathrm{kHz}$ QPO frequency vs. X-ray count rate, we show both the tracks and the branch corresponding to $\mathrm{mHz}$ QPOs showing the anti-correlation between the $\mathrm{kHz}$ QPO frequency and the X-ray count rate in Fig.1. On each correlation trackes, each data point represents the $\mathrm{kHz}$ QPO frequency and the X-ray count rate measured in 128 seconds. In the interval corresponding to the $\mathrm{mHz}$ QPOs, each data point represents that obtained in 32 seconds.

It is clearly visible that opposite to the overall positive correlation between $\mathrm{kHz}$ QPO frequency and X-ray count rate on longer time scales of hours, which forms two trackes from data on March 3, 1996 and March 6, 1996, respectively, the $\mathrm{kHz}$ QPO frequency is anti-correlated with the X-ray count rate on the interval corresponding to the $\mathrm{mHz}$ QPOs. On this $\mathrm{mHz}$ QPO interval, the $\mathrm{mHz}$ $\mathrm{QPO}$ dominates the X-ray count rate variation. Thus from the plot of $\mathrm{kHz} \mathrm{QPO}$ 
frequency vs. the X-ray count rate, we can discriminate the mHz QPO mode from that of the "parallel" tracks.

\section{Discussion and Conclusion}

Here I show that the $\mathrm{mHz}$ QPOs in $4 \mathrm{U} 1608-52$ can be identified in the plot of the $\mathrm{kHz}$ QPO frequency vs. the X-ray flux or by studying their correlation on a certain timescale corresponding to the $\mathrm{mHz}$ QPOs. This can be applied to the $\mathrm{X}$-ray variability components we know in both neutron star X-ray binaries and possibly black hole X-ray binaries. The application to the noise components and other QPOs in neutron star X-ray binaries is straight forward and we may be able to determine the origin of those variability modes.

W.Y would like to thank Prof. Michiel van der Klis for comments which has been included in the above arguments. W.Y. also would like to acknowledge partial support by the Netherlands Organization for Scientific Research (NWO) under grant 614.051.002 and partial support from NSFC (19903004). This work has made use of data obtained through the High Energy Astrophysics Science Archive Research Center Online Service, provided by the NASA/Goddard Space Flight Center.

\section{References}

Mendez, M. et al. 1998, ApJ, 505, L23

Mendez, M. 1999, astro-ph/9903469

Mendez, M. \& van der Klis, M. 1999, ApJ, 517, L51

Miller, M. C., Lamb, F. K. \& Psaltis, D. 1998, ApJ, 508, 791

Revnivtsev, M. et al. 2001, ̊̊, 372, 138

Stella, L., Vietri, M.\& Morsink, S. M. 1999, ApJ, 524, L63

Strohmayer, T. et al., 1996, ApJ, 469, L9

Titarchuk, L., Lapidus, I. \& Muslimov, A. 1998, ApJ, 499, 315

van der Klis, M. 2000, ARA\&A, 38, 717

Wijnands, R. et al. 1997, ApJ, 490, L157

$\mathrm{Yu}, \mathrm{W}$. et al. 1997, ApJ, 490, L153

Yu, W., van der Klis, M. \& Jonker, P. 2001, ApJ, 559, L29

Yu, W. \& van der Klis, M. 2002, ApJ, 567, L67

Zhang, W. et al. 1998, ApJ, 495, L9 